\title{
Selumetinib suppresses cell proliferation, migration and trigger apoptosis, G1 arrest in triple-negative breast cancer cells
}

\author{
Yan Zhou ${ }^{1+} \mathbb{D}$, Shuchen Lin ${ }^{1+}$, Kuo-Fu Tseng ${ }^{2}$, Kun Han', Yaling Wang ${ }^{1}$, Zhi-hua Gan ${ }^{1}$, Da-liu Min ${ }^{1,2^{*}}$
} and Hai-yan $\mathrm{Hu}^{1 *}$

\begin{abstract}
Background: Triple-negative breast cancer (TNBC) has aggressive progression with poor prognosis and ineffective treatments. Selumetinib is an allosteric, ATP-noncompetitive inhibitor of MEK1/2, which has benn known as effective antineoplastic drugs for several malignant tumors. We hypothesized that Selumetinib might be potential drug for TNBC and explore the mechanism.

Methods: After treated with Selumetinib, the viability and mobility of HCC1937 and MDA-MB-231 were detected by MTT, tunnel, wound-healing assay, transwell assay and FCM methods. MiR array was used to analysis the change of miRs. We predicted and verified CUL1 is the target of miR-302a using Luciferase reporter assay. We also silenced the CUL1 by siRNA, to clarify whether CUL1 take part in the cell proliferation, migration and regulated its substrate TIMP1 and TRAF2. Moreover, after transfection, the antagomir of miR-302a and CUL1 over-expressed plasmid into HCC1937 and MDA-MB-231 cell accompanied with the Selumetinib treatment, we detected the proliferation and migration again.

Results: Selumetinib reduce the proliferation, migration, triggered apoptosis and G1 arrest in TNBC cell lines. In this process, the miR-302a was up-regulated and inhibited the CUL1 expression. The later negatively regulated the TIMP1 and TRAF2. As soon as we knockdown miR-302a and over-expression CUL1 in TNBC cells, the cytotoxicity of Selumetinib was reversed.
\end{abstract}

Conclusions: MiR-302a targeted regulated the CUL1 expression and mediated the Selumetinib-induced cytotoxicity of triple-negative breast cancer.

Keywords: Selumetinib, Triple-negative breast cancer, miR-302a, CUL1

\section{Mini-Abstract}

Selumetinib inhibited the proliferation and migration of TNBC cell.

\section{Background}

Breast cancer is one of the most common cancer deaths in female. Estrogen receptor(ER)-negative breast cancer constitutes approximately $30 \%$ of breast cancer cases. Triple-negative is defined as a subgroup with ER, $\mathrm{PR}$ (progesterone receptor) and human epidermal growth

\footnotetext{
*Correspondence: mindaliu@csco.org.cn; xuri1 104@163.com

${ }^{\dagger}$ Equal contributors

'Department of Oncology, Shanghai Jiao Tong University Affiliated Sixth People's Hospital, Shanghai 200233, China

Full list of author information is available at the end of the article
}

factor receptor 2 (HER2) all negative. TNBC are assumed importance for its molecular characters, aggressive progress and distinct tranfer ability $[1,2]$. Beneficial results of current anti-HER2 or hormonal therapy could not improve the curative effect of chemotherapy. In the absence of proper treatments, TNBC often progresses to metastatic lesions in the brain and lung in three years. Once being with metastasis, the 5-year survival rate of TNBC would be less than $30 \%$. Newly therapies are urgently needed to improve the prognosis for TNBC patients. Actually, TNBCs exhibit a high level of molecular heterogeneity without high-frequency driver mutations. About $60-70 \%$ of TNBCs has mutations of p53. For PIK3CA mutations, it would be $11 \%$. No other mutations were believed as highly prevalent driver in 
TNBCs, which hampered the development of targeted therapy for TNBCs. So far, some new regimes such as anti-androgens, anti-mitotic, PI3KCA pathway inhibitors and so on, had been tested in TNBC. Here, we focus on the key survival pathway, mitogen-activated and extracellular signal-regulated kinase kinase (MEK)/extracellular signal-regulated kinase (ERK), which modulated by epidermal growth factor receptor(EGFR) [3, 4]. Overexpression of the EGFR is one of the key pathway regulating the proliferation and survive of cells. Hence, these genes may be good choose as therapeutic targets for TNBC [5]. Infante performed a phase Ib clinical study to determine the safety, tolerability, clinical activity and steady-state pharmacokinetics of trametinib, an oral MEK inhibitor, in combination with gemcitabine on breast cancer [6]. The results showed that trametinib combined with gemcitabine is safe and effective. Selumetinib, the benzimidazole ARRY-142886, has been reported to be highly potent MEK inhibitor, with an $\mathrm{IC}_{50}$ of $12 \mathrm{nmol} / \mathrm{L}$ against purified MEK [7]. There were many phase I and phase II clinical studies about Selumetinib on melanoma, colorectal cancer (CRC), non-smallcell lung cancer (NSCLC) and others, for its favorable toxicity [8-10]. Selumetinib also produces clinically meaningful increases in iodine uptake and retention in a subgroup of patients with radioiodine-refractory thyroid cancer [11]. We considered that Selumetinib might be potential drug for preventing TNBC metastasis and recurrence in a preclinical setting.

In the current study, we first found that Selumetinib inhibited proliferation and migration in two triplenegative breast cancer cell lines. Then we investigated its probable mechanism of action. MicroRNAs (miRNAs) [12] , 20-22 bp non-coding RNA, had been the hot are of cancer research for its post-transcriptional regulation function, which invovled a wide variety of biological processes, such as proliferation, differentiation, apoptosis, cell cycle and so on [13]. So far many miRs had been reported took part in MEK/ERK signaling pathway, including miR-768-3p [14], miR-221 [15], miR-199a/b-3p [16], and so on. Here, we treated TNBC cell lines HCC1937 and MDA-MB-231 with Selumetinib, and our founding indicated miR-302a/CUL1 maybe one significant downstream factors. The miR-302-367 cluster is over-expressed in embryonic stem and some kinds of carcinoma cells [17]. It works as anti-oncogene in many kinds of tumor cells, its overexpression could be of therapeutic value [18]. Kaid show that miR-302a regulated cell proliferation and self-renewal of esophageal cancer stem-like cells [19]. Here we found after treated with Selumetinib miR-302a, an well known 'bona fide' tumor suppressor, up-regulated markedly. It is difficult and costly to verified the gene regulatory networks. The bioinformatic methods offered the convenient to predicte possible diagram between miRNAs and their targets. The most publiced programs are TargetScan, miRanda, Tarbase, miRecords, RNAhybrid, and so on [20-23]. Here we found CUL-1 is the directly target of miR-302a. CUL-1 is a essential components of the p19(SKP1)/ p45(SKP2)/CUL-1 complex, named SCF, as the scaffold element [24]. Previous researches suggest that SCF as ubiquitin ligase is the key factor to cell cycle and survival. Aberrant expression of CUL-1 is critical for tumorigenesis, such as lung cancer [25], gastric cancer [26]. In the present study, we found CUL-1 also demonstrate an oncogenic activity of the OS.

\section{Methods}

\section{Cell culture and cell proliferation assay}

After planted in 96- or 6-well plates (Corning, USA) using DMEM with $10 \%$ fetal bovine serum (FBS) at $37{ }^{\circ} \mathrm{C}$ in humidified $5 \% \mathrm{CO}_{2}, \mathrm{HCC} 1937$ and MDAMB-231 cells were exposed for $24 \mathrm{~h}$ to various doses of Selumetinib(Sigma-Aldrich, Louis, MO). For the transfected process, cells were with starved in DMEM without FBS for $6 \mathrm{~h}$, then miR-302a-AMO, miR-302aMIMIC, NC or pcDNA3.1-CUL1 were added with Lipofectamine 2000 Reagent (Invitrogen) following the manufacturer's protocol. Cell proliferation assays were performed with tetrazolium salt (MTT) array according to the manufacturer's protocol.

\section{Evaluation of cell apoptosis by tunnel and FCM}

For FCM detection, the procedures were same as the cell culture previously. All cells of each group were collected and stained with Annexin V/PI following the instruction(BioVision, Palo Alto, CA, USA) The resulting was analyzed using CellQuest software (Becton Dickinson, San Jose, CA).

For tunnel test, the conditions were little different. First, cells were cultured with or without IC50 Selumetinib. For the rescue test, we first transferred the miR302a AMO or pcDNA3.1-CUL1 or the negative control for $6 \mathrm{~h}$, then we changed the medium which contain the $10 \mu \mathrm{M}$ Selumetinib. Visualized apoptotic cells were labeled with the In Situ Cell Death Detection kit (Roche) to detected positive ratio of terminal deoxytransferasemediated dUTP-biotin nick end labelling (TUNEL) following to the manufacturer's recommendations.

\section{Cell cycle analysis}

The procedure was the same as that described previously for cell culture. After washed with phosphate-buffered saline (PBS), all cells were fixed with $70 \%$ ethanol at $-20{ }^{\circ} \mathrm{C}$ for $24 \mathrm{~h}$. Then washed the cell with PBS/1 \% BSA again, stained with $30 \mu \mathrm{g} / \mathrm{ml}$ propidium iodide containing $0.25 \mathrm{mg} / \mathrm{ml}$ RNase A for $0.5 \mathrm{~h}$ in the dark, and calculated the cell cycle process ratio by FCM using Cell FIT software(Becton Dickinson, San Jose, CA). 


\section{Wound-healing assay}

In order to evaluated the motility change of TNBC cells with Selumetinib, wound healing/scratch assay was performed. TNBCs were seeded in six-well plates overnight, then scraped the confluent cell monolayer using a $200 \mu \mathrm{L}$ sterile pipette tip. After wased with PBS twice then cultured with new DMEM medium (including $10 \%$ FBS with or without IC50 Selumetinib). For the rescue test, we first transferred the miR-302a ASO or pcDNA3.1-CUL1 or the negative control for $6 \mathrm{~h}$, then we made the wound. The newly mediums contain the $10 \mu \mathrm{M}$ Selumetinib. $48 \mathrm{~h}$ later, photo images of the plates were photographed.

\section{Cell migration assay}

For the migration assay, $1.0 \times 10^{5} \mathrm{HCC}-1937$ or MDAMB-231 cells were seeded in 24-well transwell insert (pore size $8 \mu \mathrm{m}$; Corning, Inc., Corning, NY). The culture conditions were equal to above. After incubated for $12 \mathrm{~h}$, the cells adhering in the lower layer of insert were fixed and stained with $0.1 \%$ crystal violet. Photographed under light microscope at $200 \times$ magnification.

\section{Detection of differentially expressed miRNAs by miRNA} microarray

HCC-1937 cells treated with or without Selumetinib at its IC50 for $24 \mathrm{~h}$ were harvested and subsequently analyzed using a miRNA microarray (Kangcheng Biotech Company, Shanghai, China). Briefly, total miRNA was labeled, hybridized according to manufacturer's protocol. The slides were scanned by an Axon GenePix 4000B microarray scanner. Data filtering, $\log 2$ transformation, and miR normalization hot map were provided by Kangcheng Com.

\section{Targeted in vitro luciferase reporter assay}

Luciferase reporter assay using the psi-Check2 plasmid was performed as described previously to detected the interact between the miR and target [27]. The sequences used to create the wild Check2-CUL1 constructs were as follows: forward 5'-AACTCGAGGACCCGCAGCAAAT AGTTCA-3' (XhoI site in bold) and reverse 5' AATGC GGCCGCCAATGTTCAGCGTAACCCAA-3' (NotI site in bold).

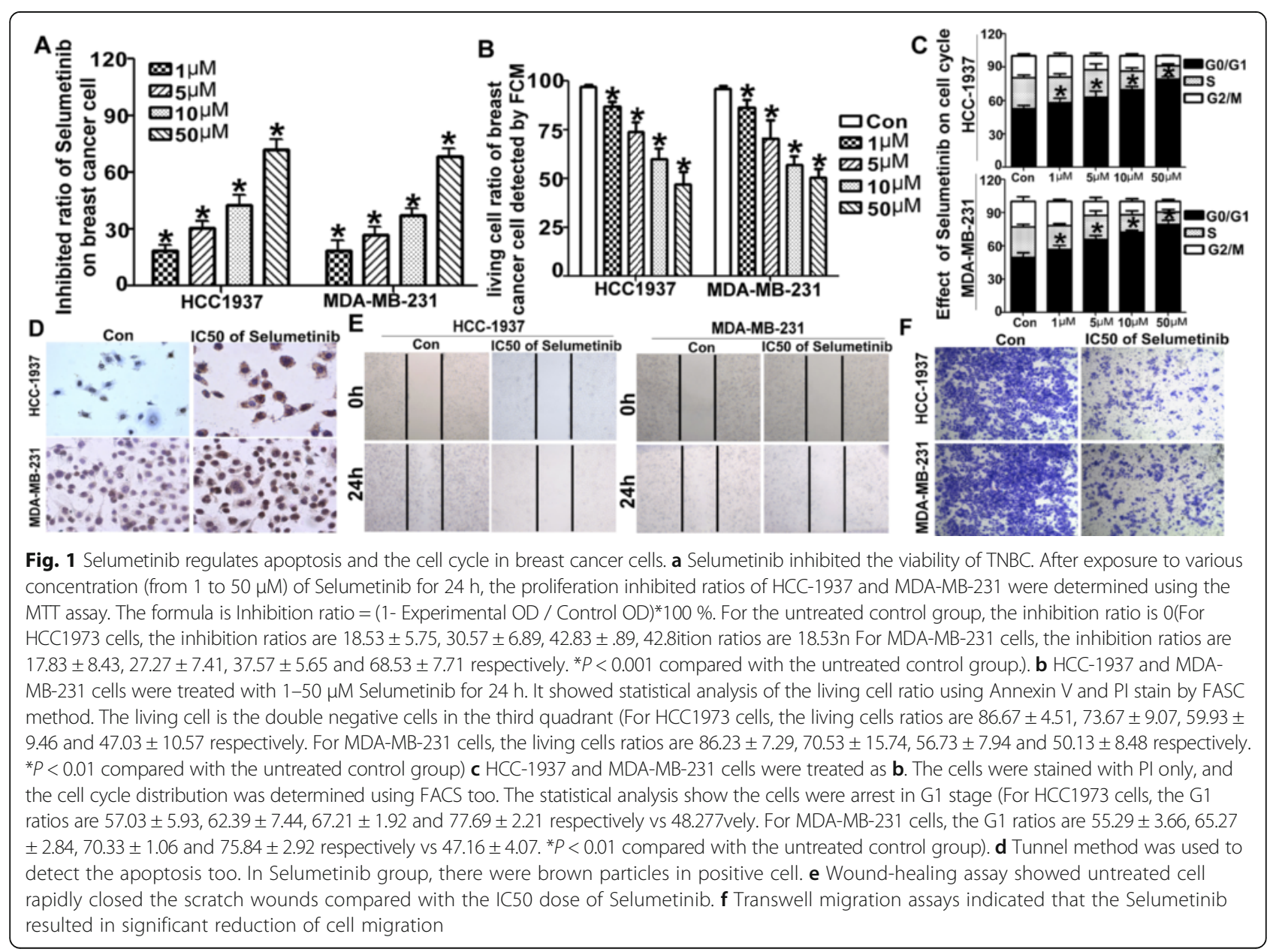




\section{Quantitative real-time PCR of miR-302a and CUL1 expression}

Total RNA of each group were abstracted with Trizol. The primer of miR-302a was purchased from Jima Com(Shanghai, China). The primers of CUL1 and its substrates TIMP and TRAF2 were as follow. The QRTPCR method was performed as described previously to detected the interact between the miR and target [27].

\begin{tabular}{lll}
\hline & Forward & Reverse \\
\hline CUL1 & 5'-GCGAGGTCCT & 5'-TTCTTCTCAATTAG \\
& CACTCAGC-3' & AATGTCAATGC-3' \\
TIMP & 5'-GCCATGGAGAGT & 5'-GCCACGAAACTGCA \\
& GTCTGCGGATACTTCC-3' & GGTAGTGCTGT-3' \\
TRAF2 & 5'GACCAGGACAAG & 5'-GCACATAGGAATC \\
& ATTGAGGC-3' & TTGGCC-3' \\
GAPDH & 5'-GAAGGTGAAGGTCG & 5'-GAAGATGGTGATGG \\
& GAGT-3' & GATTC-3' \\
\hline
\end{tabular}

\section{Western blot analysis}

The total protein were lysed in RIPA buffer and extracted. $10 \%$ SDS polyacrylamide gel was used to separated the proteins. After blocking with $5 \%$ fat-free milk for $1 \mathrm{~h}$, the membranes were incubated with antbody of CUL1 (mouse monoclonal; Invitrogen, USA), TIMP (Rabbit monoclonal; Cell Signaling Technology, MA) or TRAF2 (Rabbit polyclonal, Abcam, USA) overnight at $4{ }^{\circ} \mathrm{C}$. Blots were washed with PBST and incubated with the secondary antibody for $1 \mathrm{~h}$. Took the photo using enhanced chemiluminescence.

\section{siRNA targeting CUL1}

Designed and synthetized siRNA-CUL1(5'-CUAGAUAC AAGAUUAUACAUGCGG-3') or the control GAPDHsiRNA from GenePharma Com(Shanghai, China). The full-length CUL-1.

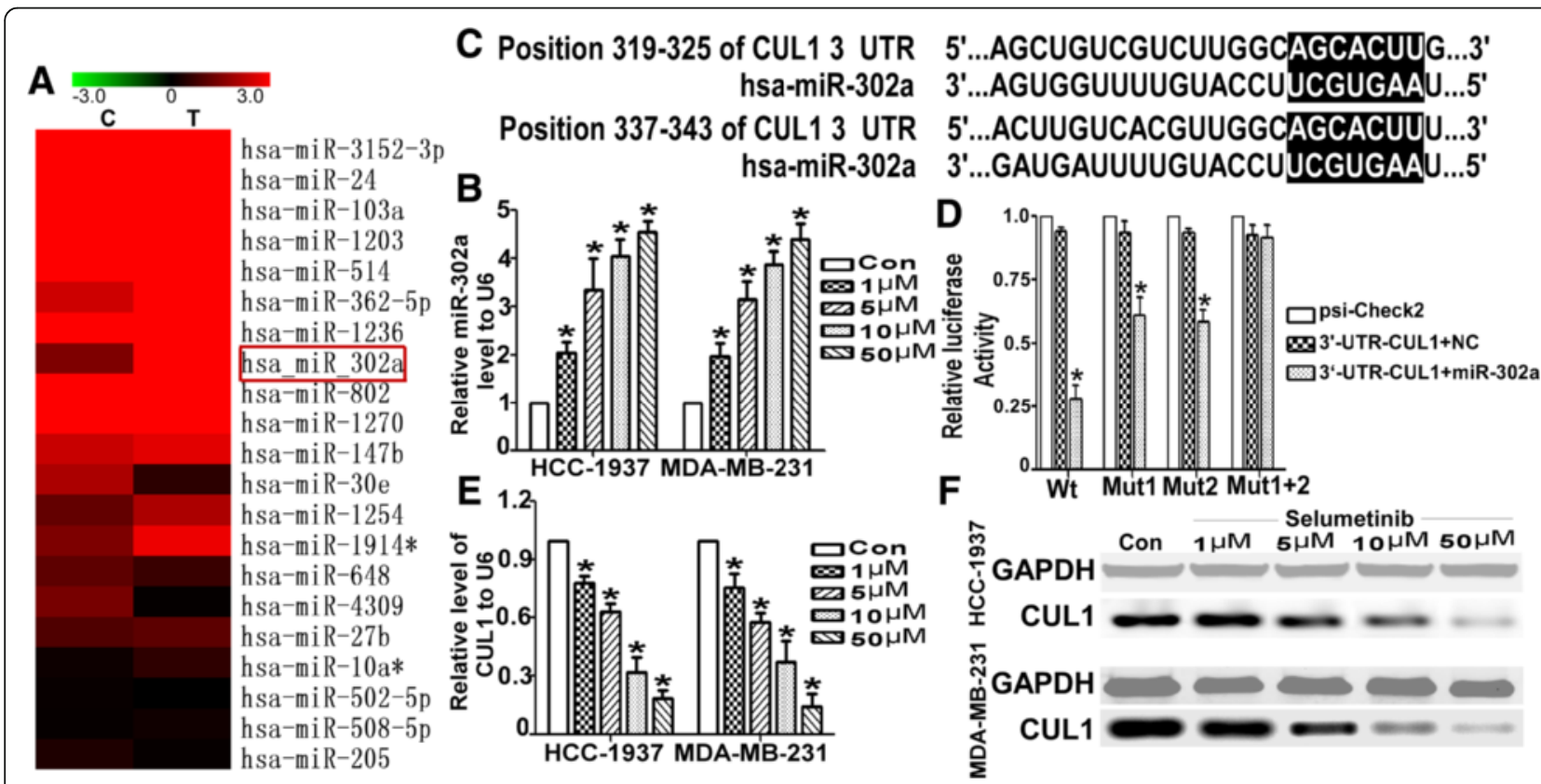

Fig. 2 Selumetinib up-regulates miR-302a and down-regulated CUL1 in TNBC cells. a Microarray analysis was used to compare the expression profiles of 703 miRNAs in HCC-1937 cells that were untreated or treated with Selumetinib. MiR-302a, one of most markedly up-regulated miRs, is labeled with a red box. $\mathbf{b}$ As detected by qRT-PCR, the miR-302a levels were dramatically increased by approximately 2- to 5-fold in the dosedependent manner in Selumetinib groups compared with the untreated group. (For HCC1973 cells, the miR-302 levels are 2.03 $\pm 0.41,3.33 \pm 1.12$, $4.03 \pm 0.61$ and $4.53 \pm 0.41$ respectively. For MDA-MB-231 cells, the miR-302 levels are $1.97 \pm 0.47,3.13 \pm 0.67,3.87 \pm 0.47$ and $4.41 \pm 0.56$ respectively. ${ }^{*} P$ $<0.01$ compared with the untreated control group, which is 1.). $\mathbf{c}$ The selection criteria of the miRNA targets were based on their common detection in the target prediction online databases as well as the full complementarity between the seed region of miR-302a and the 3'UTR of CUL1. d HEK 293 cells were co-transfected with miR-302a-MIMIC, psi-Check2, WT-psi-Check2-CUL1 or MUT-psi-Check2-CUL1. The luciferase activity levels were measured $24 \mathrm{~h}$ after transfection. The results from at least three independent experiments are presented as the means \pm SE. In this panel, the luciferase assay results show the regulation of CUL1 by miR-302a (For Wt group, after transfect the miR-302 the luciferase activity was only $0.27 \pm 0.05$. As for mutated the first seed sequence, the luciferase activity was $0.62 \pm 0.07$. As for mutated the second seed sequence, luciferase activity was $0.57 \pm 0.04 .{ }^{*} P<0.01$ compared with the control group, which is 1.). e The CUL1 mRNA level was reduced with the exposed under Selumetinib in dose-dependent manner (For HCC1973 cells, the CUL1 levels are $0.81 \pm 0.04,0.63 \pm 0.08,0.43 \pm 0.14$ and $0.23 \pm 0.07$ respectively. For MDA-MB-231 cells, the CUL1 levels are 0.78 $\pm 0.07,0.64 \pm 0.11,0.37 \pm 0.07$ and $0.24 \pm 0.05$ respectively. ${ }^{*} P<0.01$ compared with the untreated control group, which is 1.$)$. $\mathbf{f}$ The change of CUL1 protein has showed a similar trend 


\section{Construction of the CUL1 plasmid}

The CUL-1 gene was cloned into pcDNA3.1 plasmid using the primer of CUL-1 sense 5'-CAGGATCCCGTC AACCCGGAGCCAGA-3' (BamHI site in bold) and antisense 5'-AAGCGGCCGCAGAAGGGWAGCCMG$3^{\prime}$ (NotI site in bold).

\section{Results}

Selumetinib inhibited proliferation and migration in TNBC cells

Selumetinib has shown the particularly exciting therapeutic effect on many kinds of cancer. Cell proliferation was assessed in HCC1937 and MDA-MB-231 cells. Selumetinib reduced the viability ratio of both two TNBCs in dose-dependent manner (Fig. 1a). The IC50 of Selumetinib for HCC1937 and MDA-MB-231 were 15.65 and 12.94 respectively. Apoptosis and cell cycle arrest are the main reason for the inhibition of cell growth. Here we found Selumetinib trigged apoptosis and arrest of G1 stage in dose-dependent manner too (Fig. 1b, c and d). Moreover, we explored the effect of Selumetinib on cell mobility. Compared with the control group, TNBCs with IC50 of Selumetinib slowly closed the scratch wounds (Fig. 1e). he Fig. 1f showed that Selumetinib treatment led to significantly decreased in cell migration ability than the untreated control cells.

\section{Selumetinib up-regulated miR-302a and down-regulated CUL1 expression}

miRs are involved in regulating gene transcription and cell biological function. Here we detected the change of miRs in MDA-MB-231 treated with Selumetinib. miRNA array analysis showed miR-302a(sequence: GUGAAAUGUU UAGGACCACUAG) raised 3.856 times (Fig. 2a). Furthermore we verified the expression level of miR-302a was markedly and stable up-regulated in TNBCs by QRT-PCR (Fig. 2b). For miR-302a, it had been considered as a tumor suppressor [28]. A series of bioinformatics software made it easier to look for targets of miRs. miRanda show there was two combine seed sequence between miR-302a and the 3'-UTR of CUL1 (Fig. 2c). To determine whether CUL-1 is the functional target of miR-302a, we constructed WT or MUT psiR-CHECK2-CLU-1 recombinant plasmid. After transfected miR-302a-MIMIC and the plasmids in $293 \mathrm{~T}$ cells for $48 \mathrm{~h}$, the luciferase reporter activity was detected. The luciferase activity of

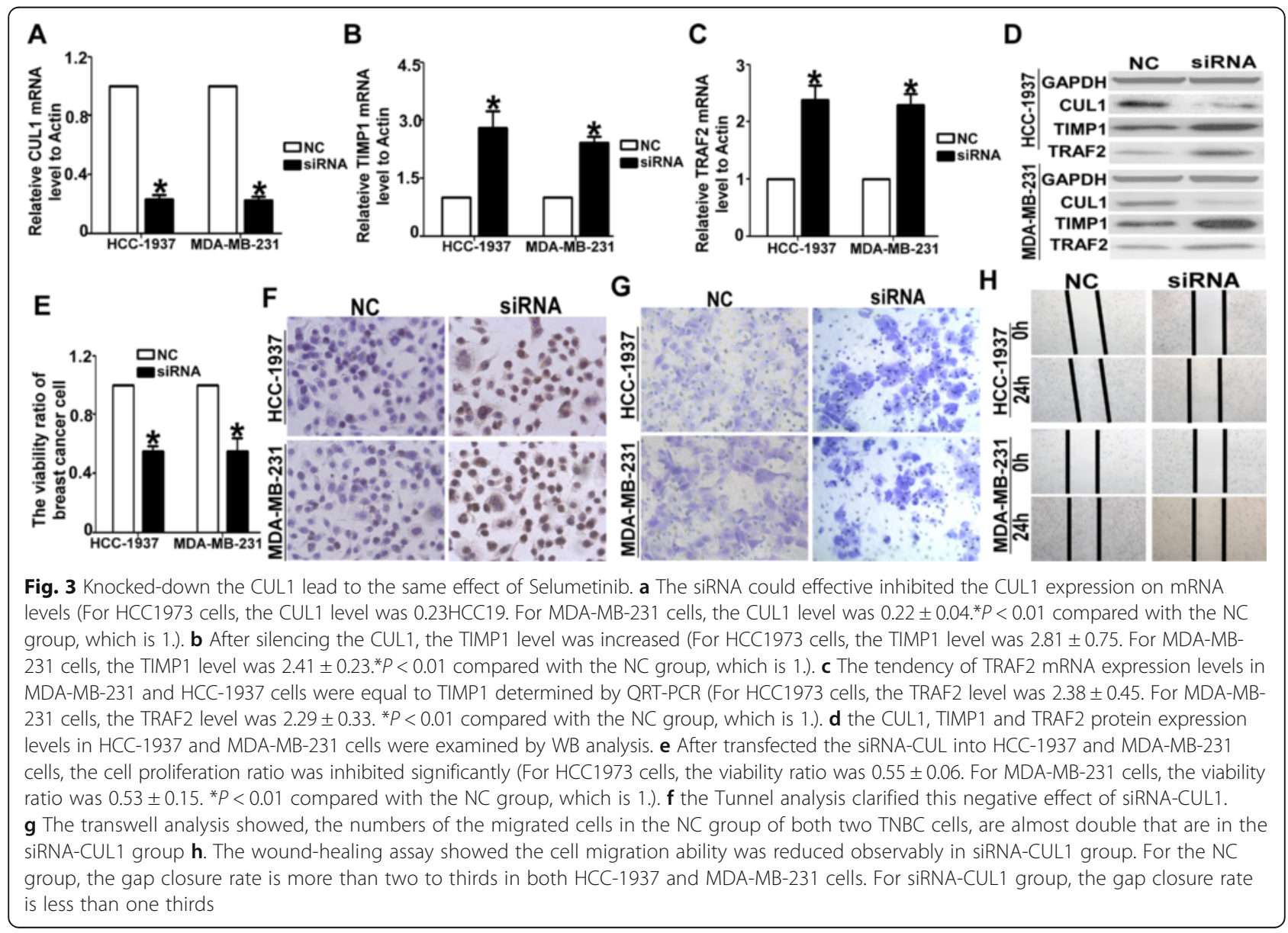




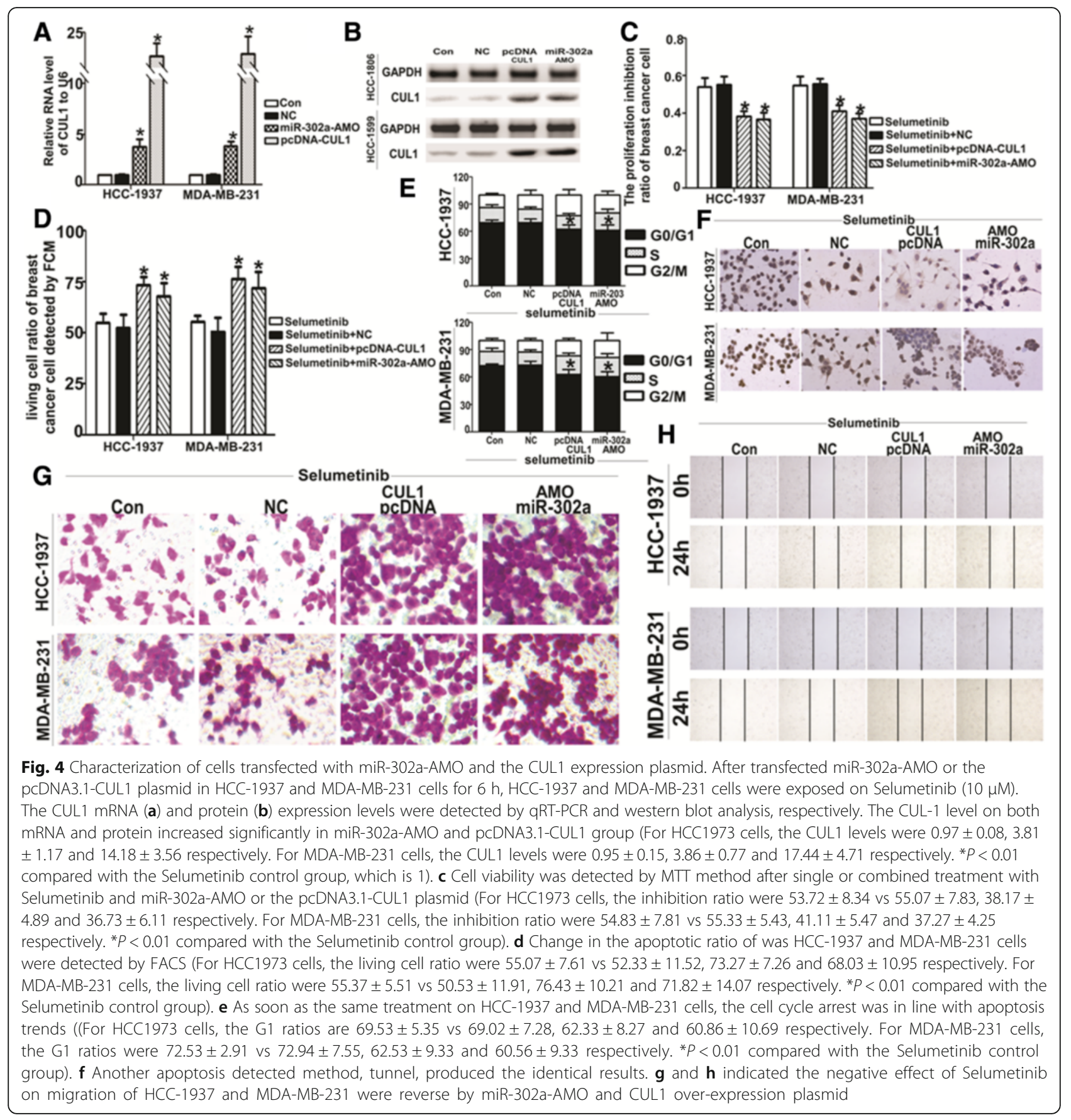

WT Check2-CUL1 was reduced to approximately 35$40 \%$ of control $\operatorname{group}(P<0.001 \mathrm{Fig} .2 \mathrm{~d})$. Conversely, there were no statistical difference between MUT group and control. What is the effect of Selumetinib on CUL-1, Fig. 2e and $\mathrm{f}$ described that Selumetinib reduced both mRNA and protein level of CUL-1 in dose-dependent manner. It is the reversely changes to miR-302a, which also proved miR-302a negative regulated CLI-1 indirectly.

\section{CUL1 regulated the degradation of key regulatory proteins}

In order to clarify the CUL1 play important role in Selumetinib on TNBC cells, we further detected the change of two downstream substrates of Cul-1, TIMP1 and TRAF2. First we knock down the CUL1 by siRNA. QRT-PCR and WB results show the siRNA effectively silencing the CUL1 expression and lead to TIMP1 and TRAF2 upregulating (Fig. 3a to d). Equal to our hypothesis, as soon 
as the CUL1 were knocked down, the cell proliferation and migration were reduced (Fig. 3e to g).

\section{Adjusted miR-302a/CUL1 level reversed the effect of Selumetinib}

In order to more deeply investigate the miR-302a/CUL-1 pathway is relevance of Selumetinib effect. We overexpressed CUL1 with pcDNA3.1-CUL1 plasmid and inhibited miR-302a with its AMO oligonucleotide, which induced up-regulating of CUL1 on both the mRNA and protein level (Fig. 4a and b). The effect of Selumetinib on TNBCs was reversed by miR-302a-AMO and pcDNA3.1CUL1 plasmid. For the inhibition of viability, in both HCC1937 and MDA-MB-231 cells, the miR-302a-AMO and pcDNA3.1-CUL1 group were 1.63- to 2.3-fold lower than other two control groups (Fig. 4c). The ratio of apoptosis and G1 stage were alos lower than two control groups (Fig. 4d to f). The Wound-healing assay and transwell test showed the migration inhibited effect of Selumetinib on HCC1937 and MDA-MB-231 were reverse by miR-302aAMO and CUL1-overexpressed plasmid (Fig. 4g, h).

\section{Discussion}

Aberrant activation of RAS/Raf/MEK/ERK signaling pathways had been reported in many kinds of cancer and been considered as targeted for its oncogenic effect. Not only pre-clinically but also many phase I or II clinical trial had been displayed the obvious therapeutic effects in solid tumor [27-29]. Some research reported that BRAF mutation was closely related to the sensitivity of Selumetinib [30]. Chen found that Selumetinib selectively rescued primary glial progenitors from TMX toxicity, such as cognitive dysfunction and changes in CNS metabolism, hippocampal volume, and brain structure, in vitro while enhancing TMX effects on MCF7 [31]. MEK pathway also plays key role in TNBC. Here, we found MAP/ERK kinase (MEK) 1/2 inhibitor, Selumetinib, repress the viability and induced apoptosis of HCC1937 and MDA-MB-231 in a dose-dependent manner. The G1 arrest and mobility declined were also linked to dose of Selumetinib (Fig. 1a to $\mathrm{d}$ ). Then we goes deeply into the mechanism. We screened miRNA profile of MDA-MB-231 with or without Selumetinib (Fig. 2a). miR-302a was significantly and gradually up-regulated miRNAs with the concentration of Selumetinib in both MDA-MB-231 and HCC-1937 (Fig. 2b). We focus on microRNA (miR)-302 family for its tumor supperessor function in many kinds of tumor [32, 33]. Yan reported miR-302 was important to miRNA-induced pluripotent stem cells (mirPS) of endometrial cancer cell lines, take part in the inhibition of cell proliferation and tumorigenicity [34]. Previous papers revealed that miR-302a regulated the expression of AKT1 [35], NR2F2 [36], CDK2 [37] and so on targeted genes. By targetscan, we predicted there were two complementary sequences in the miR-302a and 3'UTR-CUL1 (Fig. 2c). Luciferase reporter assay showed miR-302a negative regulation of CUL1 directly (Fig. 2d). According to Fig. 2e and $\mathrm{f}$, it was confirmed that CUL1 level was closed related with Selumetinib concertration. CUL1 is a key component of SCF ubiquitin ligases [38]. SCF promotes the ubiquitination and degradation of a broad range of proteins involved in cell cycle progression, signal transduction and transcription. As a key member of SCF, CUL1 is overexpressed in many kinds of cancer [39-41] and represent as target molecular for therapy [42-44]. In this paper, we found Selumetinib could inhibit both proliferation and migration in TNBC cells, and miR-302a/CUL maybe the key factor in this process. So we assume that it is equal to Selumetinib that we knocked down the CUL1 in TNBC cells. We also choose tow substrate, TIMP1 and TRAF2 of CUL1 to clarify this hypothesis. As expected, after silencing the CUL1, the viability and migration ability of TNBC cell were reduced markedly. For further prove miR-302a/CUL-1 is the operator nodes of Selumetinib onTNBCs. We regulated the miR-302 or CUL-1 level using miR-302a-AMO or CUL1 over-expression plasmid respectively in HCC1937 and MDA-MB-231 cells. Figure 4 indicated the effect of Selumetinib reversed accompany with raising of CUL-1 and silencing of miR-302a.

\section{Conclusion}

MEK pathway has been shown over-activated in TNBC. Based on our results, MEK1/2 inhibitor, Selumetinib, reduced viability through inducing apoptosis and G1 arrest, meanwhile the inhibition of mobility by Selumetinib was also be found in TNBCs. In these processes, we indicated miR-302a/CUL1 work as critical pathway in Selumetinib on TNBC.

\section{Acknowledgements \\ This study was supported by the National Natural Science Foundation of China (81072176 and 81372873). \\ This work was supported by grants from the National Natural Science Foundation of China (No 81072176 and 81372873).}

\section{Funding}

This study was supported by the National Natural Science Foundation of China (81072176 and 81372873).

\section{Availability of data and materials}

The dataset supporting the conclusions of this article is included within this article and is available from the corresponding author upon request.

\section{Authors' contributions}

DM designed research; YZ performed research; SL performed data analysis and prepared figures; KFT contributed experiments; KH provided new reagents; YW analyzed data; ZGAN polishes the English translation. HHU wrote the paper. All authors read and approved the final manuscript.

Authors' information

Not further applicable. 


\section{Competing interests}

The authors declare that they have no competing interests.

\section{Consent for publication}

Not applicable.

\section{Ethics approval and consent to participate}

Not applicable.

\section{Author details}

'Department of Oncology, Shanghai Jiao Tong University Affiliated Sixth People's Hospital, Shanghai 200233, China. ${ }^{2}$ Biophysics Department of Oregan State University, ALS-2139, Corvallis, OR 97330, USA.

\section{Received: 11 September 2015 Accepted: 8 September 2016} Published online: 21 October 2016

\section{References}

1. Griffiths $\mathrm{CL}$, Olin JL. Triple negative breast cancer: a brief review of its characteristics and treatment options. J Pharm Pract. 2012;25(3):319-23.

2. Badve S, Dabbs DJ, Schnitt SJ, Baehner FL, Decker T, Eusebi V, et al. Basal-like and triple-negative breast cancers: a critical review with an emphasis on the implications for pathologists and oncologists. Mod Pathol. 2011;24(2):157-67.

3. Liu D, He J, Yuan Z, Wang S, Peng R, Shi Y, et al. EGFR expression correlates with decreased disease-free survival in triple-negative breast cancer: a retrospective analysis based on a tissue microarray. Med Oncol. 2012;29(2):401-5.

4. Chattopadhyay C, Grimm EA, Woodman SE. Simultaneous inhibition of the HGF/MET and Erk1/2 pathways affect uvealmelanoma cell growth and migration. PLoS One. 2014;9(2):e83957.

5. Roberts PJ, Der CJ. Targeting the Raf-MEK-ERK mitogen-activated protein kinase cascade for the treatment of cancer. Oncogene. 2007;26(22):3291-310

6. Infante JR, Papadopoulos KP, Bendell JC, Patnaik A, Burris 3rd HA, Rasco D, et al. A phase $1 \mathrm{~b}$ study of trametinib, an oral Mitogen-activated protein kinase kinase (MEK) inhibitor, in combination with gemcitabine in advanced solid tumours. Eur J Cancer. 2013;49(9):2077-85.

7. Sebolt-Leopold JS, Herrera R. Targeting the mitogen-activated protein kinase cascade to treat cancer. Nat Rev Cancer. 2004;4(12):937-47.

8. Ambrosini G, Musi E, Ho AL, de Stanchina E, Schwartz GK. Inhibition of mutant GNAQ signaling in uveal melanoma induces AMPK-dependent autophagic cell death. Mol Cancer Ther. 2013;12:768-76.

9. Migliardi G, Sassi F, Torti D, Galimi F, Zanella ER, Buscarino M, et al. Inhibition of MEK and PI3K/mTOR suppresses tumor growth but does not cause tumor regression in patient-derived xenografts of RAS-mutant colorectalcarcinomas. Clin Cancer Res. 2012;18:2515-25.

10. Metro G, Chiari R, Baldi A, De Angelis V, Minotti V, Crinò L. Selumetinib: a promising pharmacologic approach for KRAS-mutant advanced non-smallcell lung cancer. Future Oncol. 2013:9(2):167-77.

11. Ho AL, Grewal RK, Leboeuf R, Sherman EJ, Pfister DG, Deandreis D, et al. Selumetinib-enhanced radioiodine uptake in advanced thyroid cancer. $\mathrm{N}$ Engl J Med. 2013;368(7):623-32.

12. Palumbo S, Miracco C, Pirtoli L, Comincini S. Emerging roles of microRNA in modulating cell-death processes in malignant glioma. J Cell Physiol. 2014; 229(3):277-86

13. Tarver JE, Sperling EA, Nailor A, Heimberg AM, Robinson JM, King BL, et al. miRNAs: Small Genes with Big Potential in Metazoan Phylogenetics. Mol Biol Evol. 2013;30(11):2369-82.

14. Jiang CC, Croft A, Tseng HY, Guo ST, Jin L, Hersey P, et al. Repression of microRNA-768-3p by MEK/ERK signalling contributes to enhanced mRNA translation in human melanoma. Oncogene. 2014;33(20):2577-88.

15. Huang F, Fang ZF, Hu XQ, et al. Overexpression of miR-126 promotes the differentiation of mesenchymal stem cells toward endothelial cells via activation of PI3K/Akt and MAPK/ERK pathways and release of paracrine factors. Biol Chem. 2013:394:1223-33.

16. Hou J, Lin L, Zhou W, Tang L, Zhou SH, Huang JP. Identification of miRNomes in human liver and hepatocellular carcinoma reveals miR-199a/ b-3p as therapeutic target for hepatocellular carcinoma. Cancer Cell. 2013; 394(9):1223-33.

17. Ge T, Yin M, Yang M, Liu T, Lou G. MicroRNA-302b suppresses human epithelial ovarian cancer cell growth by targeting RUNX1. Cell Physiol Biochem. 2014;34(6):2209-20
18. Rafiee MR, Malekzadeh Shafaroudi A, Rohban S, Khayatzadeh H, Kalhor HR Mowla SJ. Enrichment of A Rare Subpopulation of miR-302-Expressing Glioma Cells by Serum Deprivation. Cell J. 2015;16(4):494-505.

19. Kaid C, Silva PB, Cortez BA, Rodini CO, Semedo-Kuriki P, Okamoto OK. miR367 promotes proliferation and stem-like traits in medulloblastoma cells. Cancer Sci. 2015. doi:10.1111/cas.12733 [Epub ahead of print].

20. Xia W, Cao G, Shao N. Progress in miRNA target prediction and identification. Sci China C Life Sci. 2009:52(12):1123-30.

21. Witkos TM, Koscianska E, Krzyzosiak WJ. Practical Aspects of microRNA Target Prediction. Curr Mol Med. 2011;11:93-109.

22. Lewis BP, Burge CB, Bartel DP. Conserved seed pairing, often flanked by adenosines, indicates that thousands of human genes are microRNA targets. Cell. 2005;120(1):15-20.

23. Creighton CJ, Nagaraja AK, Hanash SM, Matzuk MM, Gunaratne PH. A bioinformatics tool for linking gene expression profiling results with public databases of microRNA target predictions. RNA. 2008;14(11): 2290-6.

24. Dow R, Hendley J, Pirkmaier A, et al. Retinoic acid-mediated growth arrest requires ubiquitylation and degradation of the F-box protein Skp2. J Biol Chem. 2001;276(49):45945-51.

25. Salon C, Brambilla E, Brambilla C, Lantuejoul S, Gazzeri S, Eymin B. Altered pattern of Cul-1 protein expression and neddylation in human lung tumours: relationships with CAND1 and cyclin E protein levels. J Pathol. 2007;213(3):303-10.

26. Li W, Ye F, Wang D, et al. Protein predictive signatures for lymph node metastasis of gastric cancer. Int J Cancer. 2013;132:1851-9.

27. Zhou Y, Zhao RH, Tseng KF, Li KP, Lu ZG, Liu Y, Han K, Gan ZH, Lin SC, Hu $H Y$, Min DL. Sirolimus induces apoptosis and reverses multidrug resistance in human osteosarcoma cells in vitro via increasing microRNA-34b expression. Acta Pharmacol Sin. 2016:37(4):519-29.

28. Macdonald JB, Macdonald B, Golitz LE, LoRusso P, Sekulic A. Cutaneous adverse effects of targeted therapies: Part II: Inhibitors of intracellular molecular signaling pathways. J Am Acad Dermatol. 2015;72(2):221-36.

29. Gupta A, Love S, Schuh A, Shanyinde M, Larkin JM, Plummer R, et al. DOC MEK: a double-blind randomized phase II trial of docetaxel with or withoutselumetinib in wild-type BRAF advanced melanoma. Ann Oncol. 2014;25(5):968-74.

30. Trinh VA, You Y, Hwu WJ. Treatment of BRAF-mutated advanced cutaneous melanoma. Chin Clin Oncol. 2014;3(3):28.

31. Chen HY, Yang YM, Han $R$, et al. MEK1/2 inhibition suppresses tamoxifen toxicity on CNS glial progenitor cells. J Neurosci. 2013;33:15069-74.

32. Zhang Z, Xiang D, Wu WS. Sodium butyrate facilitates reprogramming by derepressing OCT4 transactivity at the promoter of embryonic stem cellspecific miR-302/367 cluster. Cell Reprogram. 2014;16(2):130-9.

33. Wang L, Yao J, Shi X, Hu L, Li Z, Song T, Huang C. MicroRNA-302b suppresses cell proliferation by targeting EGFR in human hepatocellular carcinoma SMMC-7721 cells. BMC Cancer. 2013;13:448.

34. Yan GJ, Yu F, Wang B, Zhou HJ, Ge QY, Su J, et al. MicroRNA miR-302 inhibits the tumorigenicity of endometrial cancer cells by suppression of Cyclin D1 and CDK1. Cancer Lett. 2014;345(1):39-47.

35. Cai N, Wang YD, Zheng PS. The microRNA-302-367 cluster suppresses the proliferation of cervical carcinoma cells through the novel target AKT1. RNA. 2013:19(1):85-95.

36. Hu S, Wilson KD, Ghosh Z, Han L, Wang Y, Lan F, et al. MicroRNA-302 increases reprogramming efficiency via repression of NR2F2. Stem Cells. 2013;31(2):259-68

37. Lin SL, Chang DC, Ying SY, Leu D, Wu DT. MicroRNA miR-302 inhibits the tumorigenecity of human pluripotent stem cells by coordinate suppression of the CDK2 and CDK4/6 cell cycle pathways. Cancer Res. 2010:70(22):9473-82

38. Furstenthal L, Swanson C, Kaiser BK, Eldridge AG, Jackson PK. Triggering ubiquitination of a CDK inhibitor at origins of DNA replication. Nat Cell Biol. 2001;3(8):715-22.

39. Chen G, Li G. Increased Cul1 expression promotes melanoma cell proliferation through regulating p27 expression. Int J Onco. 2010;37(5):1339-44.

40. Bai J, Zhou Y, Chen G, Zeng J, Ding J, Tan Y, et al. Overexpression of Cullin1 is associated with poor prognosis of patients with gastric cancer. Hum Pathol. 2011:42(3):375-83.

41. Lee JG, Kay EP. Involvement of two distinct ubiquitin E3 ligase systems for p27 degradation in corneal endothelial cells. Invest Ophthalmol Vis Sci. 2008;49(1):189-96. 
42. Sun L, Shi L, Wang F, Huangyang P, Si W, Yang J, et al. Substrate phosphorylation and feedback regulation in JFK-promoted p53 destabilization. J Biol Chem. 2011;286(6):4226-35.

43. Sun L, Shi L, Li W, Yu W, Liang J, Zhang H, et al. JFK, a Kelch domaincontaining F-box protein, links the SCF complex to p53 regulation. Proc Natl Acad Sci U S A. 2009;106(25):10195-200.

44. Negi S, Kumar A, Thelma BK, Juyal RC. Association of Cullin 1 haplotype variants with rheumatoid arthritis and response to methotre xate. Pharmacogenet Genomics. 2011;21(9):590-3.

Submit your next manuscript to BioMed Central and we will help you at every step:

- We accept pre-submission inquiries

- Our selector tool helps you to find the most relevant journal

- We provide round the clock customer support

- Convenient online submission

- Thorough peer review

- Inclusion in PubMed and all major indexing services

- Maximum visibility for your research

Submit your manuscript at www.biomedcentral.com/submit 